INTEGRALS OF PRODUCTION SETS

WITH RESTRICTED SUBSTITUTION

Werner HIIDENBRAND

and

Abraham NEYMAN

$N^{\circ} 8010$ 


\title{
INTEGRALS OF PRODUCTION SETS VITTH RESTRICTED SUBSTITUTION
}

\author{
Werner Hildenbrand \\ and
}

Abraham Neyman

It has been argued in the literature, most explicitely by L.Johansen(1972), that in many applications the range of factor and/or product substitutions at the level of individual production units (plants, machines) is very limited after investment has taken place. That is to say, at a stage where capital equipments have taken form and can be considered as fixed in the short run(possibly together with other factors of production). In other words, ex post production possibility sets on the micro level are quite restricted. In the extreme case where ex post no substitutions are possible, the production possibility set of an individual production unit may be described by a segment $[0, \bar{y}]$ in the commodity space where the length of the segment is determined by the limited capacity of the production unit due to fixed capital equipments. A case might even be conceivable in which not every point on the segment $[0, \bar{y}]$ would represent a feasible production plan, for example, where the production unit can be carried out at full(normal)capacity or not carried out at all. In this extreme case the production possibility set is given by the two vectors $\{0, \bar{y}\}$. 
An industry is generally composed of many production units with different production possibility sets. Indeed, the existing production units at a given time period are, in general, of different vintages and use different techniques of production. If we exclude external effects among these production units then the total production set for the industry is obtained as the sum of the individual production sets. Thus in a model of production as explained above, i.e.,fixed input-output coefficients on the level of individual production units, one is led to study the sum or more general, the irtegral of segments ,i.e., one-dimensional convex and compact subsets in $\mathbb{R}^{n}$. Convex sets which are obtained in this way are called zonoids and have been studied in geometry (for a survey on the theory of zonoids we refer to Bolker(1969)). The theory of zonoids has recently been applied by Hildenbrand(1979) in order to study the structure of short-run industry production functions. It has been shown that the production functions which are frequently used in empirical studies(e.g. the Cobb-Douglas or CES-production functions) can not be derived within the above nociel of production. In somewhat imprecise terms there are relatively "few" production functions in the space of all production functions which can be derived in the fixed input-output coefficient model of production. Indeed it is known that the set of all zonoids in $\mathbb{R}^{\mathrm{n}}$ (i.e. integrals of 1-dimensional convex compact sets), is a closed and nowhere dense subset in the space of all compact, convex and centrally symmetric subsets of $\mathbb{R}^{n}$ where the distance of two subsets in $\mathbb{R}^{n}$ is measured by the Hausdorff distance. 
In this paper we want to examine the question whether it is decisive for this result that the summands are 1-dimensional. For example is it possible that every convex and compact subset in $\mathbb{R}^{3}$ can be obtained as an integral of two-dimensional compact and convex sets? We shall show that this again is not possible. The class of sets obtainable in this way is again closed and nowhere dense in the space of all compact and convex subsets of $\mathbb{R}^{3}$.

The study of the integral of $k$-dimensional convex compact subsets in $\mathbb{R}^{\mathrm{n}}$, where $\mathrm{k}<\mathrm{n}$, is abviously motivated by applications to production theory. Even ex post, there might be some possibility of substitution on the micro level but a restricted one compared to an ex ante consideration. This motivates to consider individual production sets which are of lower dimension that the dimension of the commodity space. In section 2 a precise formulation of the main result is given. Section 3 contains the proof. In section 4 the result is formulated in terms of production functions instead of production sets. This necessitates a consideration of the special case where there is only one output. 


\section{Definitions and the main result}

Let $K$ denote the set of compact and convex subsets of the n-dimensoinal Euclidian space $\mathbb{P}^{n}$. Me endow $K$ with the Hausdorff distance d;

$$
\mathrm{d}\left(\mathrm{K}_{1}, \mathrm{~K}_{2}\right)=\inf \left\{\varepsilon>0 ! \mathrm{B}_{\varepsilon}\left(\mathrm{K}_{1}\right) \subset \mathrm{K}_{2} \text { and } \mathrm{B}_{\varepsilon}\left(\mathrm{K}_{2}\right) \subset \mathrm{K}_{1}\right\}
$$

where ${ }^{B}{ }_{\varepsilon}(K)$ denotes the $\varepsilon$-neighborhood of $K$.

For $K \in K$ we define $\operatorname{dim} K$ as the dimension of the minimal vector subspace of $\mathbb{R}^{n}$ containing $k-k$. For $1 \leq k \leq n$, let $k_{k}=\{k \in k \mid d i m k \leq k\}$.

We denote by $L_{K}$ the set of Borel-measurable functions $h: K \rightarrow \mathbb{R}^{n}$ such that $h(K) \in K$ for every $K \in K$. Such selections always exist (e.g. see liildenbrand (1974), Lemma 1, p. 55).

Let $M\left(K_{\mathrm{k}}\right)$ denote the set of positive and bounded measures $\mu$ on (the Borel field of $k$ which are supported on $k_{k}$ (i.e. $\mu(K)=\mu\left(K_{\mathrm{k}}\right)$ ) and have the property that every function $h$ of $L_{K}$ is $\mu$-integrable. We define for every $\mu \in M\left(K_{k}\right)$

$$
\mathrm{Z}(\mu)=\left\{\int \mathrm{hd} \mu \mid \mathrm{h} \dot{\epsilon} L_{K}\right\}
$$

It is known that $Z(\mu) \in K$ (the convexity is obvious and the compactness follows, for example, From Hildenbrand (1974), Proposition $7, \mathrm{p} .73)$.

Let $z_{k}=\left\{z(\mu) \mid \mu \in M\left(K_{k}\right)\right\}$. 
Clearly $z_{k} \subset z_{k+1}$ and $z_{n}=K$.

Theorem: For every $\mathrm{k}$ with $1 \leq \mathrm{k}<\mathrm{n}$ the set $\mathrm{z}_{\mathrm{k}}$ is closed and nowhere dense in $z_{k+1}$ :

Hence, in particular, the set of compact and convex sets in $\mathbb{R}^{n}$ which cannot be obtained as an integral of compact and convex sets of dimension less than $\mathrm{n}$ is an open and dense subset in $K$. Note that sets in $Z$, are centrally symmetric. Therefore the conclusion of the Theorem is very weak for $k=1$. One can show however that the set of centrally symmetric sets in $z_{k}$ is closed and nowhere dense in the set of centrally symmetric sets in $z_{k+1}$. In view of the applications to production theory we do not restrict the sets to be centrally symmetric.

Remark: Let $(T, T, \tau)$ be a measure space of $\varphi$ a measurable compact - and convex - valued correspondence of $\mathrm{T}$ in $\mathbb{R}^{\mathrm{n}}$ which is integrable in the sense that every measurable selection of $\varphi$ is $\mu$-integrable. If $\operatorname{dim} \varphi(t) \leq k \tau$ - a.e. then the integral of the correspondence $\varphi$, which is defined as the set $\left\{\int f d \tau\right\}$ where $f$ varies over all measurable selections of $\varphi$, belongs to $z_{k}$;

$$
\int_{\mathrm{T}} \varphi \mathrm{d} \tau \in \mathrm{Z}_{\mathrm{k}}
$$

Sometimes it is more convenient to describe sets in $z_{k}$ in this way than by a measure $\mu$ in $M\left(K_{k}\right)$.

Consider a continuum of production units, i.e., an atomless measure space $(T, T, \tau)$ and an integrable correspondence $Y$ of 
$T$ in $\mathbb{R}^{n}$. If every individual production set is compact but not necessarily convex, e.g. discrete, and if $\operatorname{dim} y \leq k$ then it follows (e.g.Hildenbrand (1974) Th. 4, p.64) that

$\int \mathrm{c} \circ \mathrm{Y} d \tau=\int \mathrm{Y} d \tau$. Hence

$$
\int \mathrm{Y} d \tau \in \mathrm{Z}_{\mathrm{k}}
$$

As an application consider a continuum of production units where the production set $Y(t)$ of every production unit consists only of finitely many production plans, say two vectors $\left(a^{t}, 0, \xi_{3}^{t}, \ldots, \xi_{n}^{t}\right)$ and $\left(0, b^{t}, n_{3}^{t}, \ldots, n_{n}^{t}\right)$. Such production models with discrete choice have recently been studied by Mc Fadden (1980). Whatever will be the distribution of the input coefficients $a$ and $b$ the mean production set belongs to $z_{2}$. Thus not every production set can be obtained by aggregation of discret production sets.

\section{Proof of the theorem}

3.1. First we shall show that $z_{k}$ is a closed subset in $K$. The idea of the proof is simple; let $\left(z\left(\mu_{n}\right)\right)_{n=1} \ldots$ be a sequence in $z_{k}$ (i.e., $\mu_{n} \in M\left(k_{k}\right)$ ) which converges to $k$ in $K$. Te shall show that there is a subsequence of $\left(\mu_{n}\right)$ which "converres" to a measure $\mu$ with $z(\mu) \in Z_{k}$. Then we show that the set $z(\cdot)$ depends "continuously" on its defining measure. Hence we conclude that $\mathrm{K}=\mathrm{Z}(\mu):$ In making these arguments precise we encounter a technical difficulty since the space $k_{k}$ is not compact. However there is a compact subset $D_{k}$ of $K$, which we define below, such that to 
every measure $\mu$ in $M\left(K_{k}\right)$ correspondences a measure $v$ on $D_{k}$ such that $z(\mu)=z(\nu)+x(\mu)$. Now we can use the well-known result that a set of bounded measures on the compact space $D_{k}$ is weakly relative compact. We denote by $\rho(K)$ the maximal radius of $k \in K$, i.e.,

$$
\rho(\mathrm{K})=\max _{\mathrm{X} \in \mathrm{K}}\|\mathrm{x}\|
$$

Let $D=\{K \in K \mid \rho(K)=1$ and $0 \in K\}$ and

$$
D_{k}=\{k \in D \mid \operatorname{dim} K \leq k\} \text {. }
$$

It is not difficult to show that

(1) $D$ is a compact subset of $K$ and $D_{k}$ is closed in $D$.

Let $M(D)$ denote the set of positive and bounded measures on (the Borel subsets of) the compact space $D$. We endow $M(D)$ with the topology of weak convergence.

The following result shows that it suffices to consider measures on the compact space $D_{k}$ in order to describe the sets in $z_{k}$.

(2) For every $z \in Z_{k}$ there is a measure $\nu$ on $D_{k}$ and a vector $x \in Z$ such that

$$
z=x+z(\nu)
$$


Let $\mathrm{z} \in \mathrm{Z}_{\mathrm{k}}$. Thus there is a measure $\mu \in M\left(K_{\mathrm{k}}\right)$ such that every selection $h \in L_{K}$ is intesrable and $\mathrm{Z}=\mathrm{Z}(\mu)=\left\{\int \mathrm{h} d \mu \mid \mathrm{h} \in L_{K}\right\}$.

Let $f \in L_{K}$ and define $r(K)=\rho(K-f(K))$. It follows that $\int_{K} r(K) \mu(\mathrm{dK})<\infty$. Denote by $\eta$ the measure on $K$ which is defined by $\eta(B)=\int_{B} r d \mu$.

Let $K_{k}^{+}=\left\{K \in K_{k} \mid r(K)>0\right\}$. Then clearly $K_{k}^{+}$supports the measure $n$. Denote by $G$ the mapping of $K_{k}^{+}$into $D_{k}$ defined by

$$
G(K)=\frac{1}{r(K)}(K-f(K)) \text {. }
$$

We now define the measure $v$ on $D_{k}$ as the imace measure of $n$ with respect to the mapping $G, i . e ., \nu=n \cdot G^{-1}$. we now claim that

$$
z=x+z(\nu), \text { where } x=\int f d \mu
$$

Indeed,

$$
\begin{aligned}
K & =\left\{\int_{K} h d \mu \mid h \in L_{K}\right\}=\int_{K} f d \mu+\left\{\int_{K}(h-f) d \mu \mid h \in L_{K}\right\} \\
& =x+\left\{\int_{K_{k}^{+}} \frac{1}{r(K)}(h(K)-f(K)) \cdot n(d K) \mid h \in L_{K}\right\} \\
& =x+\left\{\int_{K_{k}^{+}} g\left(\frac{1}{r(K)} \cdot(K-f(K)) n(d K) \mid g \in L_{D_{k}}\right\}\right. \\
& =x+\left\{\int_{D_{k}} g d \mu \mid g \in L_{D_{k}}\right\}=x+z(\nu) .
\end{aligned}
$$


The following result shows that a family $\left\{\nu_{a}\right\} a \in A$ of measures on $D$ is bounded (i.e., $\sup _{A} v_{a}(D)<\infty$ ) if and only if the corresponding family $\left\{z\left(v_{a}\right)\right\}$ of subsets in $R^{n}$ is bounded.

(3) there is a constant $Q$ depending only on the dimension of IF. $^{n}$ such that for every $v$ on $D$,

$$
\rho(Z(\nu)) \leq \nu(D) \leq Q \cdot \rho(Z(\nu)) \cdot
$$

The result is well-known for zonoids, i.e., integrals of line segments, that is to say measures $v$ which are defined on $D_{1}$ (see e.r. Bolker (1960), theoren A.2.). Clearly every set $\Sigma(\nu)$, contains a zonoid. Indeed choose a measurable selection $f: D \rightarrow R^{n}$ with $f(K) \in K$ and $\|f(K)\|=1$ for every $K \in D$. Then $\int[0, f(K)] \cup(d K)$ is a zonoid which is contained in $z(v)$. This proves the second inequality. I'o prove the first, let $\psi(K, q)=\max _{x \in K}\langle x, q\rangle, K \in K, q \in \mathbb{R}^{n}$. Thus $\psi(K, \cdot)$ is the support function of $K$. Since maximazation and integration can be interchanged (see e.g. Hildenbrand (1974), Prop. 6, p.63) we obtain $\psi(z(v), q)=\int \psi(k, q) v(d K) \leq \nu(D)$. $\|q\|$ for every $q \in R^{n}$. Then it follows that $\rho(z(v)) \leq v(D)$.

Next we show

(4) the mapping $\nu \mapsto z(\nu)$ of $M(D)$ into $K$ is continuous. Let $\left(\nu_{n}\right)$ be a sequence converging weakly to $v$ in $M(D)$. vie have to show that the seruence $\left(z\left(\nu_{n}\right)\right)$ converges in 
the Hausdorff distance to $Z(v)$.

Since $\nu_{n}(D) \rightarrow \nu(D)$ it follows from (3) that the sequence $\left(\rho\left(z\left(\nu_{n}\right)\right)\right)$ is bounded. By a well-known result (often called Blaschke's selection theorem) there exists a subsequence of $\left(Z\left(\nu_{n}\right)\right)$ converging to a set $z \in K$. Thus we may assume that $z\left(\nu_{n}\right) \rightarrow z$. One easily verifie: that this implies $\psi\left(z\left(v_{n}\right), q\right) \rightarrow \psi(z, q)$ for every $q \in R^{n}$. For every $q \in R^{n}, \psi(\cdot, q): D \rightarrow R$ is a bounded continuous function. Hence

$\psi\left(z\left(\nu_{n}\right), q\right)=\int \psi(K, q) \nu_{n}(d K) \rightarrow \int \psi(K, q) v(d K)=\psi(z(v), q)$ for every $q \in R^{n}$. Thus it follows that $\psi(z, q)=\psi(z(v), q)$ for every $q \in R^{n}$, which implies that $z=z(v)$.

Finally we shall show that

(5) $z_{k}$ is closed in $K$.

Let $\left(z_{n}\right)$ be a sequence in $z_{k}$ converging to $z \in k$. By (2) there are $x_{n} \in z_{n}$ and measures $\nu_{n}$ on $D_{k}$ such that $z_{n}=x_{n}+z\left(\nu_{n}\right)$.

Since the sequence $\left(x_{n}\right)$ is bounded we can assume without loss of generality that $\left(x_{n}\right) \rightarrow x$. Thus $z\left(\nu_{n}\right)=z_{n}-x_{n}$ converges to $K-x$. Hence $\left(\rho\left(z\left(\nu_{n}\right)\right)\right)$ is bounded and consequently by (3) the sequence $\left(\nu_{n}\left(D_{k}\right)\right)$ is bounded. Since $D_{k}$ is compact there exists a subsequence of $\left(\nu_{n}\right)$ converging weakly to a measure $\nu$ on $D_{k}$. By the continuity of $\nu \rightarrow Z(v)$. it follows that $z=x+Z^{\prime}(v)$. Since $v$ is a measure on $D_{k}$ we obtain $z \in Z_{k}$. 
3.2. In this section we shall show that

(6) if $\mathrm{n} \geq 3$ and $\mathrm{k}<\mathrm{n}$ then for every $\mathrm{Z} \in \mathrm{Z}_{\mathrm{k}}$ there is a $\mathrm{k}+1$ dimensional simplex $\Delta$ such that $\mathrm{z}+\alpha \Delta \notin \mathrm{Z}_{\mathrm{k}}$ for every $\alpha>0$.

Since $\tilde{i}+\alpha \Delta \in z_{k+1}$ this clearly implies that $z_{k}$ is nowhere dense in $z_{k+1}$.

A compact convex subset $K$ of $\mathbb{R}^{n}$ is called indecomposable if for any decomposition of the form $K=K_{1}+K_{2}$ with $k_{i} \in K \quad(i=1,2)$ there are $x_{1}, x_{2}$ in $\mathbb{R}^{n}$ and $\beta_{1}, \beta_{2}$ in $\dot{\mathbb{R}}_{+}$such that $\mathrm{K}_{i}=\mathrm{x}_{i}+\beta_{i} \mathrm{~K} \quad(i=1,2)$.

For example, every k-dimensional simplex is indecomposable (Grünbaum (1967) p.318).

We shall need the following result which we shall prove in section 3.3 .

Proposition 1: If the integral j $\varphi \mathrm{d} \tau$ of a compact- and convex- valued measurable.correspondence $\varphi$ of the measure space $\left(T^{\prime}, T, \tau\right)$ into $\mathbb{R}^{n}$ is indecomposable then there are intecrable functions $x: T \rightarrow \mathbb{R}^{n}$ and $\beta: T \rightarrow \mathbb{R}_{+}$such that

$$
\varphi(t)=x(t)+\beta(t) \cdot \int \varphi d \tau \cdot \tau-a \cdot e \cdot
$$

and $\int B$ d $\tau=1$. 
Lemma 1: If the face $F(Z, q)$ of $Z \in Z_{\mathrm{l}}$ with normal $q$ is indecomposable and $\operatorname{dim} F(Z, q)=k$ then the face $F(Z, q)$ is a summand of $\mathrm{Z}$.

Proof: Since $Z \in Z_{k}$ there is a measure space $(T, T, \tau)$ and an integrable correspondence $\varphi$ of $T$ into $K$ with $\operatorname{dim} \varphi(t) \leq k$ such that $z=\int \varphi d T$. It is known that the face of an integral of a correspondence is equal to the integral of the faces of the correspondence (e.g. Hildenbrand (1974), p. 65). Thus

$$
F(z, q)=\int F(\varphi(t), q) \tau(d t) .
$$

Since the face $F(z, q)$ is indecomposable there are by Proposition 1 integrable functions $\mathrm{x}: \mathrm{T} \rightarrow \mathbb{R}^{\mathrm{n}}$ and $\dot{\mathrm{B}}: \mathrm{T} \rightarrow \mathbb{R}_{+}$ with $\int B d t=1$ such that $\tau$ - a.e.,

$$
F(\varphi(t), q)=x(t)+B(t) F(z, q)
$$

Let $T_{1}=\{t \in T \mid B(t)>0\}$. Then for $\tau-$ a.e. $t$ in $T_{1}$ $F(\varphi(t), q)$ is a translate of $F(z, q)$. Since $\operatorname{dim} \varphi(t) \leq k$ and $\operatorname{dim} F(z, q)=k$ we conclude that, for $\tau-$ a.e. in $T_{1}$,

$$
\varphi(t)=F(\varphi(t), q)
$$

Let $\varphi\left\{\mathrm{T}_{1}\right\}=\int_{\mathrm{T}_{1}} \varphi \mathrm{d} \tau$ and $\quad \mathrm{x}\left\{\mathrm{T}_{1}\right\}=\int_{\mathrm{T}_{1}} \mathrm{xd} \tau$. Then

$$
\varphi\left\{T_{1}\right\}=\int_{T_{1}} F(\varphi(t), q) \tau(d t)=x\left\{T_{1}\right\}+F(z, q)
$$

Thus $F(Z, q)$ is a summand of $Z$ since

$$
\mathrm{z}=\int_{\mathrm{T} \backslash \mathrm{T}_{1}} \varphi \mathrm{d} \tau+\mathrm{x}\left\{\mathrm{T}_{1}\right\}+\mathrm{F}(\mathrm{z}, \mathrm{q}) . \quad \text { Q.E.D. }
$$


Lemma 2: If the face $F(K, q)$ of $K \in K$ with normal $q$ is a summand of $K$ then $F(K, q)$ is a summand of the face $F(K,-q)$ with normal $-q$; hence

$$
\operatorname{dim} F(K,-q) \geq \operatorname{dim} F(K, q)
$$

Proof: One easily verifies that

$$
F(F(K, q),-q)=F(K, q) .
$$

Then we obtain for $K=Q+F(K, q)$ that

$$
\begin{aligned}
F(K,-q) & =F(Q+F(K, q),-q)=F(Q,-q)+F(F(K, q),-q) \\
= & F(Q,-q)+F(K, q) .
\end{aligned}
$$

Thus $F(K, q)$ is a summand of $F(K,-q)$ Q.E.D.

We now proceed to proof assertion (6).

Let $\mathrm{k}<\mathrm{n}$ and $\mathrm{z} \in \mathrm{Z}_{\mathrm{k}}$.

There exists $q \in R^{n}, q \neq 0$, such that the face $F(z, q)$ of $z$ with normal $q$ and the face $F(z,-q)$ of $z$ with normal -q consist each of one point, i.e.,

$$
\operatorname{dim} F(z, q)=\operatorname{dim} F(z,-q)=0
$$

Indeed, let $\mathrm{q}=\overline{\mathrm{x}}_{2}-\overline{\mathrm{x}}_{1}$, where $\overline{\mathrm{x}}_{1}$ and $\overline{\mathrm{x}}_{2}$ are defined by $\left\|\bar{x}_{2}-\bar{x}_{1}\right\|=\max \left\{\left\|x_{1}-x_{2}\right\| \mid x_{1}, x_{2} \epsilon z\right\}$.

Further there is a k+1-dimensional simplex $\Delta$ such that for every $\alpha>0$ the face $F(\alpha \Delta, q)$ is a k-dimensional simplex 
while the face $F(\alpha \Delta,-q)$ contains just one point, i.e., $\operatorname{dim} F(\alpha \Delta,-q)=0$. Indeed, choose $x_{0}=-q, x_{1}, \ldots, x_{k}$ to be orthogonal vectors and let $\Delta$ be the convex hull of $\left\{x_{0}, \ldots, x_{k}\right\}$

Let $K=z+\alpha \Delta$. We shall show that $K \notin z_{k}$. Assume to the contrary that $k \in Z_{k}$. By definition of the vector $q$ and the simplex $\Delta$ it follows that $\operatorname{dim} F(k,-q)=0$ and that $F(K, q)$ is a translate of the $k$-dimensional simplex $\alpha \Gamma(\Delta, q)$.

But every $k$-dimensional simplex is indecomposable, and therefore by Lemma 1 it follows that $F(K, q)$ is a summand of $K$. But then we obtain by Lemma 2 that

$$
0=\operatorname{dim} F(K,-q) \geq \operatorname{dim} F(K, q)=k ;
$$

a contradiction.

\subsection{Proof of Proposition 1}

First we shall prove the following

Lemma 3: Let $\varphi$ be a compact-and convex-valued measurable correspondence of $(T, T, \tau)$ into $\mathbb{R}^{n}$. Let $K \in K$ and assume that for every $S \in T \int_{S} \varphi \mathrm{d} \tau=\alpha \mathrm{K}$ for some $\alpha>0$. 
Then there is a measurable function $B: T \rightarrow \mathbb{R}_{+}$such that $\varphi(t)=\beta(t) K \quad \tau$-almost everywhere and $\int \beta d \tau=1$ whenever $\operatorname{dim} K \geq 1$

The case $\operatorname{dim} \mathrm{K}=0$ is a standard result. If $\operatorname{dim} \mathrm{K} \geq 1$ there is $q \in \mathbb{R}^{\mathrm{n}}$ with $\psi(\mathrm{k}, \mathrm{q})=1$. Define the function $B$ by

$$
B(t)=\psi(\varphi(t), q)
$$

For $y \in \mathbb{R}^{n}$, let

$$
\begin{aligned}
& \mathrm{T}_{0}=\{t \in \mathrm{T} \mid \psi(\varphi(t), y)=\beta(t) \psi(K, y)\} \\
& \mathrm{T}_{+}=\{t \in \mathrm{T} \mid \psi(\varphi(t), y)>B(t) \psi(K, y)\} \\
& \mathrm{T}_{-}=\{t \in \mathrm{T} \mid \psi(\varphi(t), y)<B(t) \psi(K, y)\}
\end{aligned}
$$

If $\tau(T(y, t))>0$ then

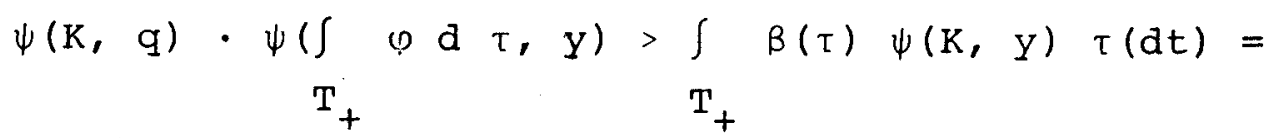

$$
\begin{aligned}
& \int \psi(\varphi(t), q) \psi(K, y) \tau(d t)=\psi(K, y) \cdot \psi\left(\int \varphi d \tau, q\right), \\
& \mathrm{T}_{+} \mathrm{T}_{+}
\end{aligned}
$$

which leads to a contradiction with the assumption $\int_{T} \varphi d \tau=\alpha \mathrm{K}$.

Thus $\tau\left(T_{+}\right)=0$. Analogously one shows that $\tau\left(T_{-}\right)=0$. Hence, for any given $y \in \mathbb{R}^{n}$,

$$
\psi(\varphi(t), y)=\beta(t) \cdot \psi(K, y) \cdot \tau-a \cdot e .
$$


Let $\left\{y_{i}\right\}_{i=1}, \ldots$ be a dense countable subset of $\mathbb{R}^{\mathrm{n}}$. Then, $\tau$-almost everywhere

$$
\psi\left(\varphi(t), y_{i}\right)=\beta(t) \psi\left(K, y_{i}\right) \quad, i=1, \ldots .
$$

Using the continuity of the support function we conclude that for $\tau$-almost every $t$

$$
\psi(\varphi(t), y)=\beta(t) \psi(K, y) \text { for every } y \in \mathbf{R}^{n},
$$

which implies that $\varphi(t)=\beta(t) K \quad \tau-$ a.e.

\section{Proof of Proposition 1:}

For $K$ in $k$ let $s(K) \leq K$ denote the steiner point of $K$. (For a definition see, e.g., Grünbaum (1967), chapter 14). Recall if $\varphi$ is a compact- and convex- valued measurable correspondence then

$$
s\left(\int \varphi d \tau\right)=\int s \circ \varphi d \tau
$$

Thus, if $K=\int \varphi d \tau$, then $\hat{K}=\int \hat{\varphi} d \tau$ where $\hat{K}=K-s(K)$ and $\hat{\varphi}(t)=\varphi(t)-s(\varphi(t))$.

If $K$ is indecomposable and if $K_{1}$ is a summand of $k$ with $s(K)=s\left(K_{1}\right)=0$ then $K_{1}=\alpha K$ for some $\alpha \geq 0$. Since for $\mathrm{S} \subset \mathrm{T}$ the integral $\int \hat{\varphi} \mathrm{d} \tau$ is a summand of $\hat{\mathrm{K}}$ and since

$$
s\left(\int_{S} \hat{\varphi} d \tau\right)=\int_{S} s(\varphi(t)-s(\varphi(t)) \tau(d t)=0=s(\hat{K})
$$

we conclude from Lemma 1 that there is a function, $\beta: T \rightarrow \mathbb{R}_{+}$ with $\int \beta d \tau=1$ such that

$$
\hat{\varphi}(t)=\beta(t) \hat{K} \quad \tau-a \cdot e .
$$




$$
\text { Hence } \varphi(t)-s(\varphi(t))=\beta(t)(K-s(K)) \quad \tau \text { - a.e. }
$$

Let $x(t)=s(\varphi(t))-\beta(t) s^{\prime}(K)$. Thus the functions $B$ and $\mathrm{x}$ have the desired properties.

\section{Production Functions}

To every compact and convex subset $Y$ of $\mathbb{R}^{r_{1}}$ we associate a concave function $F_{Y}$.

Let $D_{Y}$ denote the projection of $Y$ on the space of the first $\mathrm{n}-1$ coordinates of $\mathbb{R}^{\mathrm{n}}, \mathrm{i} \cdot \mathrm{e}$.

$$
D_{Y}=\left\{\left(x_{1}, \ldots, x_{n-1}\right) \in \mathbb{R}^{n-1} \mid\left(x_{1}, \ldots, x_{n-1}, x_{n}\right), Y \text { for some } x_{n} \in \mathbb{R}\right\}
$$

The function $F_{Y}: D_{Y} \rightarrow \mathbb{R}$ is defined by

$$
F_{Y}\left(x_{1}, \ldots, x_{n-1}\right)=\max \left\{x_{n} \in \mathbb{R} \mid\left(x_{1}, \ldots, x_{n}\right) \in Y\right\}
$$

If the first $\mathrm{n}-1$ coordinates are integrated as inputs and the $n$ - th coordinates as output then we call $F_{Y}$ the efficient production function associated to the production set $Y$.

Let $D$ be an open and $G$ a compact subset in $\mathbb{R}^{n-1}$ with $D \subset G$. For every $k$ with $1 \leq k \leq n$ we denote by $F_{k}(D, G)$ the class of all production functions $F_{z}$ which are associated with some set $z \in Z_{k}$ with $0 \in Z$ and. $D \subset \operatorname{proj}_{\mathbb{R}^{n-1}} \mathrm{Z} \subset \mathrm{G}$. 
Clearly $F_{n}(D, G)=F(D, G)$ equals the set of all bounded concave functions defined on $D$ with $F(0)=0$.

For $n=2$ one easily verifies that $F_{1}(D, G)=F(D, G)$.

Theorem: If $n>2$ and $k<n$ then $F_{k}(D, G)$ is closed and nowhere dense in $F(D, G)$ with respect to both the pointwise and uniform convergence topologies.

This result does not follow directly from the theorem in section 2 but it can be proved with similar techniques. 


\section{$\underline{\text { References }}$}

Bolker, E.D., (1969): "A Class of Convex Bodies" Transactions of the Amer. Math. Soc. 145, 323-344.

Grünbaum, B., (1967): Convex Polytopes, Interscience Publishers London, New York, Sydney.

Hildenbrand, W., (1979): "Short-Run Industry Production Functions Based on Microdata" to appear in Econometrica.

McFadden, D., (1980): "Econometric Models of Probability Choice" in C. Manski and D. MCFadden (eds)

Structural Analysis of Discrete Data

M I T Press, Cambridge. 